\title{
Macro-structure et spécialité dans les textes de sciences humaines
}

Pascale Fade

\section{(2) OpenEdition}

\section{Journals}

Édition électronique

URL : http://journals.openedition.org/asp/4050

DOI : 10.4000/asp.4050

ISSN : 2108-6354

\section{Éditeur}

Groupe d'étude et de recherche en anglais de spécialité

\section{Édition imprimée}

Date de publication : 1 décembre 1994

Pagination : 121-127

ISSN : 1246-8185

\section{Référence électronique}

Pascale Fade, « Macro-structure et spécialité dans les textes de sciences humaines », ASp [En ligne], 5-6 | 1994, mis en ligne le 06 décembre 2013, consulté le 09 mai 2019. URL : http:// journals.openedition.org/asp/4050 ; DOI : 10.4000/asp.4050

Ce document a été généré automatiquement le 9 mai 2019.

Tous droits réservés 


\title{
Macro-structure et spécialité dans les textes de sciences humaines
}

\author{
Pascale Fade
}

Aux derniers colloques du GERAS à Montpellier et à Grenoble, j'ai présenté le fascicule Compréhension écrite en anglais pour les sciences humaines (1992), qui s'adresse aux étudiants de $2^{\text {e }}$ année de DEUG Sciences humaines à Nancy 2. Ce fascicule développe la méthodologie de lecture élaborée par deux des membres de l'équipe d'enseignants aux spécialistes d'autres disciplines que les langues, méthodologie fondée en particulier sur les notions d'enchaînement et de structure du texte.

2 Je prendrai ici comme point de départ ces notions pour étudier plus précisément comment se manifeste l'organisation textuelle au niveau macro et j'évoquerai quelques applications pédagogiques. J'analyserai ensuite le lien éventuel entre ces deux facettes et la spécialité même.

\section{Organisation textuelle au niveau macro}

\section{Organisation textuelle}

3 Si un texte est considéré comme une unité sémantique, c'est qu'il existe une relation sémantique continue entre les différents éléments qui le constituent. Cette relation sémantique est établie par l'utilisation de procédés discursifs qui confèrent au texte à la fois sa cohésion et sa cohérence.

4 Rappelons en quelques mots que la cohésion fait référence aux liens formels qui existent entre les phrases afin d'assurer le développement propositionnel du discours ; c'est-à-dire qu'il existe en surface des marqueurs explicites grammaticaux et lexicaux qui assurent la continuité du discours.

5 La cohérence, elle, fait référence à la structure du discours tant sur le plan logique que sur le plan de l'organisation du contenu propositionnel. Les différents éléments qui composent le discours ne se présentent pas de façon aléatoire mais sont organisés, et ce 
choix dans l'organisation dépend du raisonnement tenu par l'auteur, du message qu'il veut faire passer mais aussi du respect des conventions spécifiques associées à chaque type de discours. La cohérence, à l'inverse de la cohésion, n'implique pas nécessairement la présence de marqueurs explicites, les relations étant alors déduites du contexte.

6 S'il est possible d'étudier les phénomènes de cohésion à partir du développement phrase à phrase d'un texte il est aussi possible de s'intéresser à l'organisation textuelle au niveau macro et de vérifier comment se réalisent alors ces relations.

\section{Niveau macroscopique du texte}

7 Dans notre méthodologie de lecture (élaborée d'après notre corpus d'articles), nous insistons sur le fait qu'après la lecture de l'introduction d'un texte, qui permet de découvrir le thème de celui-ci, la lecture en continu de la première phrase de chaque paragraphe permet de découvrir sa structure et le thème développé dans chacune des parties. La première phrase revêt donc une importance particulière car elle contient un ou plusieurs liens qui la mettent directement en relation avec le contexte précédent et qui confèrent au paragraphe un statut particulier à la fois dans la hiérarchie des informations et dans la structure du discours. Lorsque la première phrase d'un paragraphe ne présente aucun marqueur explicite qui la relie au contexte qui précède, cette phrase annonce le début d'une nouvelle partie.

Ceci est toutefois une simplification de la réalité car rares sont les ruptures franches et une continuité existe bel et bien dans tout le texte. Je prends Halliday et Hasan pour référence :

Discourse does not wander at random from one topic to another but runs on reasonably systematic lines with a certain consistency of topic and predictability of development.

9 La première phrase d'un paragraphe pourra donc être considérée comme l'amorce d'une nouvelle partie car elle présente une idée ou une information nouvelle tout en présentant également un lien logique au niveau de la structure macroscopique du texte. Il est tout aussi vrai qu'au niveau du contenu, une référence au contexte global pourra être présente sans empêcher pour autant le démarrage d'une nouvelle partie.

\section{Cohésion au niveau macro}

Dans le corpus considéré, textes de la grande presse à orientation sciences humaines, c'est l'organisation du discours au niveau macroscopique et en particulier certains phénomènes qui participent à la cohésion que je vais envisager ici. Je laisse volontairement de côté des procédés comme l'énumération ou la suite temporelle du simple fait que leurs marqueurs sont flagrants et le rôle de ceux-ci rapidement identifiable. Le lecteur, même moyen, les repère aisément et leur attribue leur rôle sans difficulté.

11 Je vais plus particulièrement m'intéresser à la grande classe des anaphoriques. Cette étiquette pour moi recouvre l'ensemble des mots et expressions qui reprennent une partie de texte quelle qu'elle soit ( $\mathrm{du}$ simple mot à un ensemble de paragraphes) et ceux dont la présence présuppose obligatoirement d'autres éléments en amont du discours auxquels ils référent. Le comparatif et le superlatif, par exemple, appartiennent à cette 
catégorie car ils présupposent nécessairement d'autres éléments antérieurs avec lesquels s'établit la relation et auxquels il faut se référer pour prétendre à une véritable compréhension du texte.

Sur le plan grammatical, on trouve l'ensemble des pronoms, les expressions introduites par un déterminant démonstratif, possessif, indéfini, numéral (...), les pro-formes de substitution (one, do, so), l'ellipse, le comparatif, le superlatif...

13 Sur le plan lexical, on trouve la simple répétition, l'utilisation de synonymes, de génériques et de termes appartenant au même champ sémantique. Pour ces derniers, il ne s'agit pas strictement de termes de reprise, mais la co-occurrence de contraires, de complémentaires ou de termes ayant une relation lexico-sémantique particulière n'est pas innocente et la deuxième occurrence doit obliger le lecteur à établir un lien avec l'occurrence qui précède.

Je ne vais pas reprendre systématiquement l'ensemble de ces deux listes, mais évoquer en particulier ceux dont la fréquence est élevée ou ceux qui posent problème au lecteur.

\section{Étude de quelques anaphoriques}

\section{This/these - That/those}

$\mathrm{Au}$ niveau macroscopique, on remarque la très forte présence de this/these, tant en pronom qu'en déterminant, et il est clair que leur rôle est (quasiment) toujours anaphorique. La distribution this cataphorique vs that anaphorique semble totalement tombée et l'emploi de this/these avec des référents non personnels est très fortement majoritaire, la notion de proximité ou non-proximité du référent n'intervenant pas. Les rares apparitions de that nous conduisent même à penser qu'il s'agit d'un emploi marqué, d'une forme d'insistance de la part de l'auteur. En revanche, avec un référent personnel, la répartition these/those apparaît équilibrée et liée à la proximité ou non du référent ( these : proche, those : éloigné). Dans l'article de Jean-Louis Vidalenc (1993), j'ai été surprise de découvrir que cette opposition puisse poser problème dans la traduction et la rédaction d'articles scientifiques. J.L. Vidalenc écrit :

Nous avons rencontré plus haut un This qui est anaphorique en contexte. Cette divergence avec le comportement habituellement décrit dans les grammaires de ce marqueur est bien connue des traducteurs chevronnés qui ne sont pas, sur le terrain, pris au dépourvu par ce problème. Leur intuition de la langue leur permet de trouver une solution satisfaisante au coup par coup.

Si cette question gêne les étudiants de CAPES et leurs enseignants, tiraillés entre grammaires de référence et usage évolutif de la langue, elle nous conduit toutefois à nous interroger sur les divergences qui peuvent exister entre les différents types de discours et sur la réalisation concrète de certains phénomènes, en apparence simples et bien circonscrits. Dans le cas qui nous intéresse, l'emploi d'anaphoriques correspond à une nécessité dans le discours, quel que soit le discours, et la nature même du discours ou sa spécialité ne semble pas, a priori, déterminante dans le choix de tel ou tel anaphorique. Les problèmes soulevés par J.L. Vidalenc dans l'enseignement/apprentissage de la rédaction d'articles scientifiques doivent encourager une plus grande coopération entre spécialistes des différentes aptitudes à l'intérieur d'une même discipline : il est clair que les observations et les études dans le domaine de la compréhension écrite doivent avoir un impact sur l'enseignement/apprentissage de l'expression.

\section{Le comparatif et le superlatif}



paragraphe : leur rôle dans l'organisation du discours est principalement d'annoncer une information ou un argument supplémentaire à d'autres déjà cités. Le superlatif arrive évidemment souvent en fin de liste et son rôle est effectivement un rôle de clôture. Le comparatif peut aussi avoir ce rôle de clôture mais on ne peut en être sûr qu'en abordant le paragraphe suivant.

Exemple $1:$ "Trapped in the ice" (International Management, March 1982)

Dans cet exemple,"The newest, and most controversial, reason" pourrait très bien être remplacé par the last ou the final reason. On perdrait bien sûr ce qu'apportent les adjectifs new et controversial au développement mais on voit que le superlatif confère à cette raison un rôle de clôture dans la partie.

Exemple $2:$ «How to protect abused children » (Newsweek, November 23, 1987)

$\S$ :Identifying a case of child abuse is only the first step, and even placing the child

in foster care isn't always the answer. [...]

$\S$ :Many child-care professionals say the answer lies in better efforts to keep troubled families together $-[. .$.

$\S$ : Short-term intervention is also helpful. [...]

$\S$ :Of course, prevention is the best cure for child abuse - [...]

est cure a pour rôle d'annoncer la dernière réponse possible au problème des enfants maltraités. Tout en ne sachant pas le nombre exact de réponses qui vont être présentées, the best cure ferme obligatoirement la liste. D'autant plus qu'ici, les réponses sont clairement données dans un ordre croissant d'efficacité ou de qualité.

\section{Les anaphoriques lexicaux}

En première phrase de paragraphe, the suivi d'un terme générique a très fréquemment une valeur anaphorique, the pouvant être assimilé à un démonstratif. Cette caractéristique de l'article défini étant bien connue et bien documentée, je me limiterai à ce simple rappel. En revanche, je voudrais insister sur le rôle que jouent les items lexicaux qui appartiennent au même champ lexico-sémantique. Même si le terme d'anaphorique ne les englobe pas strictement, la co-occurrence de ces items, dans un contexte défini, fait que chacun d'entre eux reprend une partie du thème annoncé et on peut alors, dans cette optique, leur attribuer une valeur anaphorique. D'un paragraphe à l'autre, on va retrouver, en première phrase, des items qui font référence à un même thème et lui donnent corps. C'est ainsi que le thème annoncé au début d'une partie va se trouver confirmé d'un paragraphe à l'autre, mais sans pouvoir, a priori, prévoir le choix lexical. D'autres marqueurs accompagnent souvent ces items lexicaux, mais ils ne sont pas obligatoires pour percevoir l'unité d'une partie.

Exemple : "The Modern Ark" (The Economist, July 9, 1983)

$\S:$ Whether zoos should play a role in conserving rare animals from extinction is controversial. [...]

$\S$ :Mr Gerald Durrell, the writer and founder of Jersey Zoo, was an early proponent of the argument that zoos can provide insurance against rare animals going extinct in the wild. [...]

$\S$ :The pros and cons of whether captive breeding can help an endangered species are illustrated by [...]

$\S$ : There are two camps. [...]

$\S$ : In the 1960s, the "hands-off" lobby called the tune.

$\S$ : In 1981, [...]; so now, once again, the "hands-on" lobby is back in charge.

Pour conclure, je citerai à nouveau Halliday \& Hasan : 
It is not a case of there being particular lexical items which always have a cohesive function. EVERY lexical item MAY enter into a cohesive relation, but by itself it carries no indication whether it is functioning cohesively or not. That can be established by reference to the text.

\section{Applications pédagogiques}

Lorsque nous demandons à un étudiant, qui a assimilé notre méthodologie de lecture, de souligner dans la première phrase de chaque paragraphe d'un texte les liens qui existent d'un paragraphe à l'autre, il va, sans trop d'hésitation, repérer ces liens. Toutefois, quand ce lien est un anaphorique, même s'il est bien repéré en tant que tel, l'identification de son référent reste au mieux imprécise et au pire inexistante. Le référent d'un all this, par exemple, va immanquablement être identifié comme étant « tout ce qui précède dans le texte ", même si des indices indiquent un référent plus limité et plus précis.

Il apparaît indispensable en étudiant la structure d'un texte, de demander systématiquement le référent de tout anaphorique rencontré si l'on veut s'assurer qu'une véritable compréhension s'instaure. Cela peut sembler fastidieux à l'étudiant, mais doit provoquer par la suite chez lui une sorte de réflexe conditionné. Lorsqu'il se trouvera en situation de lecture autonome, ce à quoi nous le préparons, il effectuera cette recherche par automatisme.

Deux exercices me semblent particulièrement appropriés et efficaces pour faire prendre conscience aux étudiants de l'importance et du rôle des anaphoriques :

L'exercice qui consiste à remettre en ordre les différents paragraphes d'un texte donnés dans le désordre présente le gros avantage de «forcer » le lecteur à trouver le référent des divers anaphoriques présents s'il veut réussir la tâche entreprise. Il prend conscience que l'anaphorique n'a de sens véritable qu'une fois mis en relation avec ce qu'il reprend et que la phrase dans laquelle il se situe n'a de sens qu'une fois l'anaphorique explicité. D'autre part, le caractère ludique de cet exercice est un plus non négligeable dans l'apprentissage.

La création de vrai/faux où la proposition de référent pour un anaphorique est erronée peut aussi contribuer à la réflexion sur le procédé, car cet exercice conduit le lecteur à vérifier la justesse de ses hypothèses et à démontrer leur validité.

Enfin, il n'est pas inutile non plus d'attirer l'attention des étudiants sur le caractère parfois obscur de ce qu'ils disent en cours, dans leur langue maternelle, à cause de l'utilisation d'anaphoriques sans référent explicite ou globalement à cause d'un discours réduit qui laisse une forte place à l'implicite. Ils considèrent, a priori, que l'on sait de quoi ils parlent, mais il est bon quelquefois de s'assurer que leurs références et les nôtres sont effectivement semblables.

Ceci nous conduit directement à envisager les liens qui existent entre l'organisation du discours et le contexte situationnel d'une part et la spécialité d'autre part.

\section{Organisation du discours et spécialité}

9 Je viens de faire référence aux étudiants qui présupposent que nous savons de quoi ils parlent étant donné le contexte. Cette situation peut être transposée à l'auteur d'un texte qui présuppose certaines connaissances à son lecteur. 

caractéristiques du lecteur supposé, le type d'article qu'il écrit, le sujet même de l'article. Chacune de ces variables va l'influencer dans le choix des informations et dans l'organisation du contenu.

Tout d'abord, si le but de l'auteur est bien d'être lu, il va particulièrement s'attacher à ce que son texte soit adapté au lecteur potentiel tel qu'il l'imagine et aux attentes de celui-ci. Dans le cadre d'une spécialité, pour le lecteur visé, l'auteur va définir en particulier un domaine de référence qu'il considère commun à lui-même et au lecteur. On peut donc prévoir que dès que les caractéristiques du lecteur ne seront plus celles du profil envisagé, ce domaine de référence sera inadéquat. Ceci est de première importance pour nous enseignants, quelle que soit la spécialité de nos étudiants. Lorsque nous choisissons un texte de spécialité, nous devons nous interroger sur les connaissances requises, dans la spécialité, pour le comprendre. Le risque est de proposer l'étude d'un texte pour lequel, ne partageant pas le domaine de référence, le lecteur ne disposera pas des outils nécessaires à sa compréhension. En particulier, les relations non explicites établies entre deux informations risquent fort de n'être pas perçues. J'illustre ce propos par un commentaire d'Elizabeth Crosnier (1993) sur la cohérence des abstracts scientifiques :

Les auteurs font preuve d'une grande économie en ce qui concerne l'articulation des notions. Ils s'adressent à un public de spécialistes qui comprennent immédiatement l'enchaînement et l'ordre des idées sans la nécessité de les formaliser. Le «non-encore » spécialiste ou futur spécialiste va, lui, juger le texte incohérent, car il
n'arrive pas à déceler la logique qui sous-tend le discours. La cohérence n'est donc pas
une notion absolue mais bien une notion relative qui dépend du type de lecteur pour un
texte donné. Le «non-encore » spécialiste ou futur spécialiste va, lui, juger le texte incohérent, car il
n'arrive pas à déceler la logique qui sous-tend le discours. La cohérence n'est donc pas
une notion absolue mais bien une notion relative qui dépend du type de lecteur pour un
texte donné. discours. En témoignent les nombreuses publications qui s'intéressent aux difficultés liées à la rédaction d'articles scientifiques : notamment celles de Jean-Louis Vidalenc (1993) déjà cité, de Ray Cooke (1993), ou encore les articles qui concernent la description d'un type particulier de discours, comme le travail ci-dessus mentionné d'E. Crosnier (1993) sur les abstracts scientifiques. Il existe bel et bien des conventions et des normes qui doivent être respectées pour chaque type de discours faute de quoi le texte est jugé non conforme et donc irrecevable. Jean-Louis Vidalenc fait référence à des articles refusés par des referees: " articles sans fautes sur le plan de la correction lexicale et grammaticale, mais qui n'étaient pas structurés selon les critères habituels de leur revue ». Et Ray Cooke insiste également sur le fait qu'un article doit, afin d'avoir des chances d'être publié, répondre aux exigences de ce qu'il décrit ainsi : «what might loosely be termed the 'good communication practice' of scientific and technical English ».

34 À la question posée dans son introduction «Peut-on dire par exemple qu'il y a un style canonique propre à l'abstract?» Elizabeth Crosnier apporte une réponse prudente dans sa conclusion : "Il est difficile de parler de forme canonique, du moins en informatique » mais ajoute aussitôt que l'« on retrouve des constantes, à part quelques exceptions, dans tous les textes ».

Il convient donc, dans notre enseignement, de familiariser les étudiants avec les conventions qui régissent les types de discours qu'ils sont amenés à rencontrer, car, comme l'écrit Widdowson (1978) : 
If we are not familiar with the conventions, then the language is incoherent to a

degree corresponding with our unfamiliarity.

Les conventions et les normes sont donc liées au type de discours et s'y s'ajoutent parfois les conventions propres à telle ou telle revue. Plus important encore : il est fait allusion plusieurs fois à l'évolution de ces conventions, évolution qu'il est indispensable de mettre à jour si l'on veut coller à la réalité du terrain.

Le troisième facteur, le sujet même de l'article, influence aussi l'organisation textuelle. Dans notre corpus, de nombreux textes sont informatifs et, selon le cas, rapportent une enquête, montrent l'évolution d'un fait de société, démontrent une tendance, expliquent un phénomène. Tous ces articles ont en commun que l'auteur y fait en quelque sorte « le tour de la question » et épuise le sujet. Cette caractéristique commune s'accompagne fréquemment de l'organisation textuelle suivante, quelle que soit la provenance de l'article: le texte débute par un exemple (ou une série d'illustrations) qui va ensuite donner lieu à l'exposition du thème général du texte. L'auteur ensuite, selon le cas, expose les faits, les étapes ou les arguments qui se rapportent au thème ou qui le constituent. Le texte va fortement s'appuyer sur le "déjà dit » pour progresser, ce qui explique certainement la présence très fréquente d'anaphoriques en première phrase de paragraphe. La conclusion, elle, offre une grande variété : elle peut être ouverte ou fermée, ou être l'occasion pour l'auteur de se manifester par un jugement, un commentaire ou une mise en garde.

8 Il est difficile de dégager le rôle précis de la culture de la discipline sur l'organisation textuelle. Toutefois, on peut estimer que l'histoire de la discipline, le fait qu'elle soit récente ou non, qu'elle soit partagée par un grand nombre ou non, qu'elle soit considérée noble ou non, qu'elle soit reconnue ou non ont tous un impact sur la rhétorique du discours. Les conventions et les normes évoquées précédemment sont une des manifestations de cette culture mais le linguiste est-il en mesure de définir avec exactitude et précision la culture d'une discipline sans en être lui-même un spécialiste?

Si le linguiste constate des différences entre le discours de diverses disciplines, il peut les attribuer à la culture propre à chacune d'entre elles, mais il ne pourra pas, sans l'aide du spécialiste, établir une liaison entre la spécificité du discours et la spécificité de la culture.

Pour conclure, j'insisterai à nouveau sur la coopération indispensable entre les différents spécialistes du discours, quel qu'il soit, pour que les observations et analyses faites par les uns et les autres soient profitables à tous. D'autant que les règles évoluent et que la sagacité de chacun est donc nécessaire pour mettre à jour les nouvelles tendances.

\section{BIBLIOGRAPHIE}

Billant J. et Fade P. 1992. Compréhension écrite en anglais pour les sciences humaines. Nancy : Presses universitaires de Nancy.

Cooke R. 1993. « Learning to publish in English: how can French researchers bridge the gap ? . ASp 1, 463-474. 
Crosnier E. 1993. «L'abstract scientifique anglais-français : contraintes et libertés ». ASp 2, 177-198.

Fade P. 1993. «L'anglais de spécialité pour non-spécialistes niveau Deug ». ASp 1, 287-300.

Halliday M.A.K. \& Hasan R. 1976. Cohesion in English. Londres : Longman.

Vidalenc J. 1993. « Un apport de l'analyse du discours à l'apprentissage de la rédaction d'articles scientifiques : l'étude des phénomènes de connexion ». ASp 1, 383-401.

Widdowson H.G. 1978. Teaching Language as Communication. Oxford : Oxford University Press.

\section{RÉSUMÉS}

Cet article décrit comment se manifeste l'organisation textuelle au niveau macroscopique et le rôle de certains anaphoriques en particulier. Les liens entre organisation du discours et spécialité sont analysés dans une perspective pédagogique.

Discourse organisation at a macroscopic level and the appearance of some specific anaphoric items are studied. The relation between discourse organisation and the subject matter is analysed from a pedagogical angle.

INDEX

Keywords : cohesion, macro-structure, reading comprehension

Mots-clés : anaphorique, compréhension écrite, macro-structure

\section{AUTEUR}

\section{PASCALE FADE}

Pascale Fade enseigne à l'Université Nancy 2. <pascale.fade@univ-lorraine.fr 